\title{
Clustering between HMXBs and OB associations in the Milky Way
}

\author{
Arash Bodaghee* \\ UC Berkeley - Space Sciences Laboratory \\ E-mail: bodagheedssl.berkeley.edu
}

John A. Tomsick

UC Berkeley - Space Sciences Laboratory

E-mail: jtomsickessl.berkeley.edu

\section{Jérôme Rodriguez}

CEA Saclay

E-mail: jrodriguez@cea.fr

\section{J. Berian James}

UC Berkeley - Lawrence Berkeley Laboratory

E-mail: bjames@lbl.gov

We present the first direct measurement of the spatial cross-correlation function of high-mass X-ray binaries (HMXBs) and active OB associations in the Milky Way. This result relied on a sample containing 79 hard X-ray detected HMXBs (mostly IGRs) and 458 OB associations. Clustering between the two populations is detected with a significance above $7 \sigma$ for distances $\leq 1 \mathrm{kpc}$. Thus, HMXBs closely trace the underlying distribution of the massive star-forming regions that are expected to produce the progenitor stars of HMXBs. The average minimum separation of $0.4 \pm 0.2 \mathrm{kpc}$ between $\mathrm{OB}$ associations and HMXBs is consistent with the view that the latter have large runaway velocities $\left(100 \pm 50 \mathrm{~km} \mathrm{~s}^{-1}\right)$. This suggests that the offset is mostly due to the runaway velocity acquired by the binary during and after the supernova phase (with a few exceptions). The characteristic scale of the correlation function indicates an average kinematical age (三 time between the supernova and X-ray phase) of $4 \pm 2 \mathrm{Myr}$ for the HMXB population. Despite being derived from the "grand design" of our Galaxy, these signatures of HMXB evolution are consistent with theoretical expectations and observations of individual objects.

An INTEGRAL view of the high-energy sky (the first 10 years) - 9th INTEGRAL Workshop and celebration of the 10th anniversary of the launch

15-19 October 2012

Bibliotheque Nationale de France, Paris, France

\footnotetext{
${ }^{*}$ Speaker.
} 


\section{Introduction}

High-mass X-ray binaries (HMXBs) are systems in which a compact object (usually a neutron star) accretes from a massive stellar companion $\left(M \gtrsim 10 M_{\odot}\right)$. The mass-age relation in stellar evolution predicts that $\sim 10^{7} \mathrm{yr}$ elapses between starbirth and supernova in high-mass stars [1]. Thus, HMXBs are relatively young systems which are not expected to migrate far from their birthplaces: sites with a recent history of massive star formation, i.e., the OB associations (OBAs) that trace the Galactic spiral arms [2]. A precise study of the spatial relation of massive star-forming regions and the HMXBs they spawn can offer valuable insight into stellar and Galactic evolution.

Observational evidence linking Galactic HMXBs and OBAs has been demonstrated in individual cases in which the connection is attested by other factors: i.e., consistent proper motions and distances [3]. While INTEGRAL has been instrumental in helping to increase the known population of HMXBs, the evidence on a Galactic scale is limited to comparing distributions of longitudes or galactocentric distances $[4,5,6,7,8]$. The drawback is that a single spatial dimension is considered at a time, and so precious information about the true proximity of objects in the populations is lost. A better approach is to construct the spatial (or cross-) correlation function $\xi[9,10]$.

Our sample includes all INTEGRAL-ISGRI detected sources that are confirmed or strongly suspected of being HMXBs. For each HMXB, we searched through the literature and extracted the most recent distance measurement and related uncertainty when available. We strove to consider only distances that were derived from observations of the optical/IR counterpart, and not X-ray luminosity derived distances nor those assumed from, e.g., being in the line of sight of a particular spiral arm or the Galactic Center (GC). Any HMXB suspected of or confirmed to belong to the Magellanic Clouds was excluded. This resulted in 79 Galactic HMXBs whose distances are known (Fig. 1). The positions and distances of 458 OBAs are drawn from [11].

\section{The Spatial Correlation Function}

For a given $\mathrm{HMXB}$ in a volume element $\delta V_{\mathrm{HMXB}}$, the probability $\delta P$ (in excess of Poisson) of finding an OBA in a volume element $\delta V_{\mathrm{OBA}}$ separated by a distance $r$ (in kpc) is:

$$
\delta P=n_{\mathrm{HMXB}} n_{\mathrm{OBA}}[1+\xi(r)] \delta V_{\mathrm{HMXB}} \delta V_{\mathrm{OBA}}
$$

The mean number density of each population is given by $n_{\mathrm{HMXB}}$ and $n_{\mathrm{OBA}}$. We assume that the displacement along the $z$-axis is negligible (which we confirmed via other means), and so surface rather than volume elements are used.

A catalog is generated containing a list of OBAs whose locations in the Galaxy have been randomly distributed. Rings of 1-kpc thickness (from 0 to $20 \mathrm{kpc}$ ) are constructed around each HMXB. In each ring, we count the number of observed OBAs, and we keep a separate tally of the number of OBAs drawn from a random distribution. An HMXB-OBA (observed) pair is referred to as $D D$ (for data-data), while an HMXB-OBA (randomized) pair is labelled $D R$ (for data-random). The number densities of the observed and randomized distributions are $n_{D}$ and $n_{R}$, respectively. In this way, we construct $\xi$ for each radius according to the definition of [9]:

$$
\xi(r)=\frac{n_{R} D D}{n_{D} D R}-1
$$




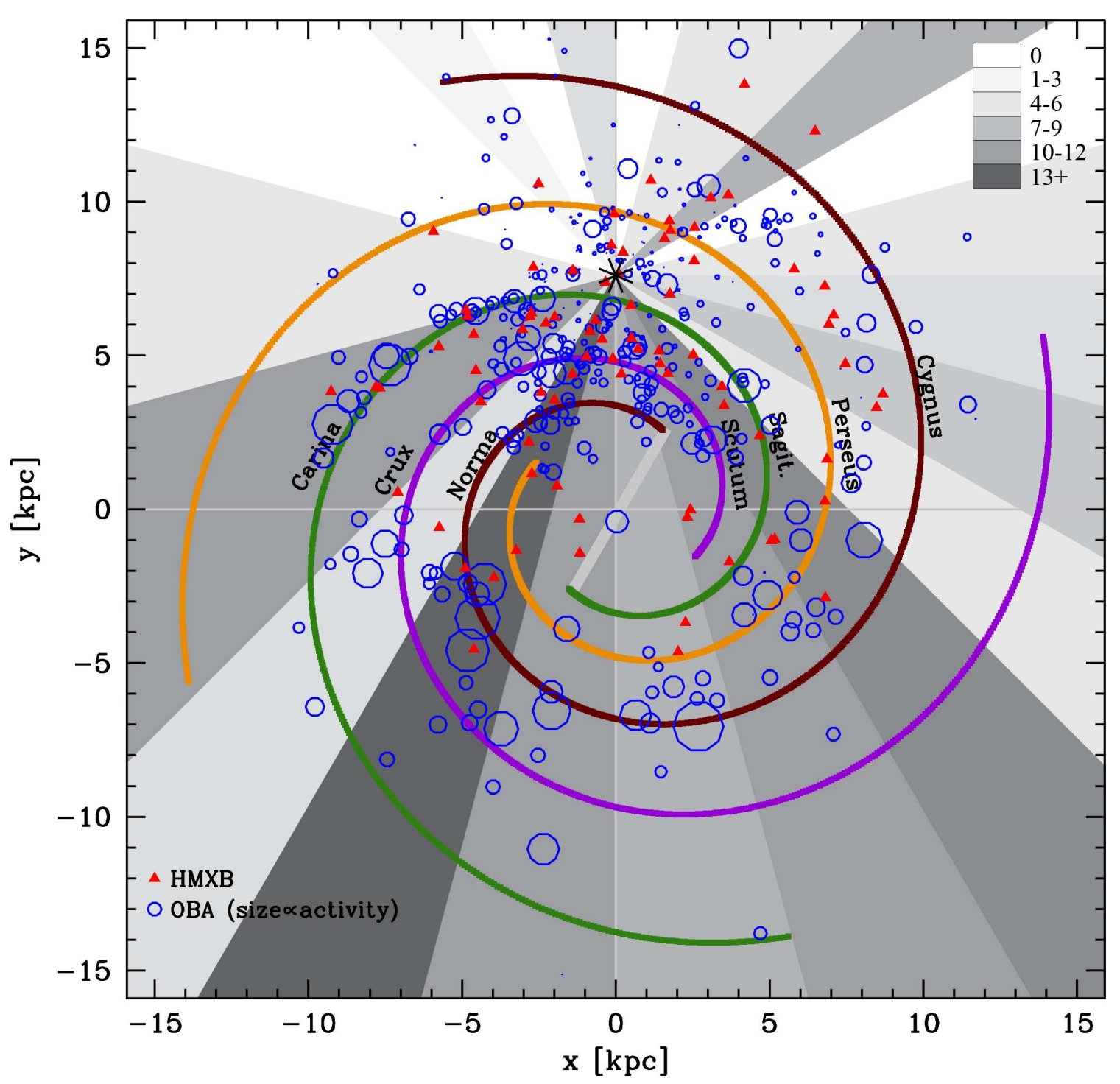

Figure 1: The Milky Way as viewed from above showing the locations of 79 INTEGRAL-detected HMXBs whose distances are known, and the spiral arm model of [12]. The shaded histogram displays the number of HMXBs (including those without distances) in each $15^{\circ}$ longitude bin. The HMXB concentration peaks towards the tangents to the Inner Perseus/Norma and Scutum/Sagittarius arms which contain many active stellar nurseries called OB associations (OBAs: 458 blue circles, with symbol size proportional to the amount of stellar activity in the complex: from [11]).

If $\xi=0$, which implies that each ring contains as many $D D$-pairs as $D R$-pairs, then Eq. 2.1 is simply a uniform Poissonian probability. However, if $\xi>0$, then there is a higher chance of an HMXB having a neighbor that is an observed (rather than a randomized) OBA. Several types of random distributions were considered, but we settled on the one that is most similar to the OBA distribution: a Gaussian ring centered at $7.6 \mathrm{kpc}$ from the $\mathrm{GC}$ with $\sigma_{z}=2.5 \mathrm{kpc}$. One thousand trials were performed and the average $\xi$, along with its $1-\sigma$ uncertainties, are shown in Fig. 2. 


\section{Results \& Biases}

Our measured value of $\xi(r<1 \mathrm{kpc})=1.61 \pm 0.22$ implies that around a given HMXB, one counts between 2 and 3 times as many observed OBAs as randomized ones. For $r<3 \mathrm{kpc}$, the clustering signal is $7-11 \sigma$ in excess of Poisson. The observed and random surface-density distributions are statistically compatible only at large radii (i.e. $r \gtrsim 5 \mathrm{kpc}$ ). In other words, HMXBs and OBAs are clustered together.

Observational biases can affect the correlation function. For example, an HMXB situated far from the Sun needs to be correspondingly more luminous in order to be detected in the X-rays. Large distances also make the optical classification as an HMXB more difficult: line spectroscopy of the donor star is hindered by reddening and absorption from interstellar material in the Galactic Disk. This leads to a preponderance of HMXBs (as well as observed OBAs) close to the Sun, and a paucity of such objects situated behind the GC and its bar. We tested this effect by restricting the analysis to objects located within an 8-kpc radius around the Sun-a perimeter inside of which most HMXBs and OBAs should be detectable and their distances known with reasonable accuracy. When only pair counts of objects in the Solar neighborhood are considered, the clustering signal at small radii persists with a statistical significance of $7 \sigma$ for $r<1 \mathrm{kpc}$. This suggests that omitting the effects of interstellar extinction has a negligible impact on our conclusions.

Some HMXBs have distances with uncertainties that are on the order of, or larger than, the $1-k p c$ spatial binning that we used. The counts per ring will fluctuate depending on the distance precision quoted in the literature. To determine the impact of this bias, we shuffled each HMXB according to a Gaussian profile within its line-of-sight distance range as defined by its error bars. When an error bar was not provided in the literature, we set the uncertainty range to be equal to the average of the known error bars of the sample $(1.5 \mathrm{kpc})$. Then, we regenerated $\xi$ by randomly shuffling the HMXB distributions within their line-of-sight uncertainties. The clustering signal is above $7 \sigma$ in all trials for $r<3 \mathrm{kpc}$ from a given HMXB. This indicates that despite the large uncertainties that plague some of our objects, the clustering signal that we measure for the populations remains significant.

We tested the correlation between HMXBs and OBAs against the spiral arm model of [11] whose equations represent the best-fitting four-arm logarithmic spiral to the locations of OBAs. Here, we adopted the Sun-GC distance of $8.5 \mathrm{kpc}$ assumed in the model. Figure 2 shows that there is no significant correlation between OBAs and the spiral arms with $\xi(r) \sim 0$ for all $r$ (i.e., $D D \sim D R$ for all $r$ ). Similarly, we found no significant clustering between HMXBs and the points representing the spiral arms. Restricting the analysis to objects in the Solar vicinity (i.e., within $8 \mathrm{kpc}$ of the Sun) leads to similar conclusions, so we can rule out an apparent lack of distant OBAs (or HMXBs) skewing the results. In other words, around any given OBA (or HMXB), one tends to find as many neighbors drawn from a randomized Gaussian ring distribution, as neighbors representing the spiral arms. These comparisons of our observed stellar samples with the Galactic models allow us to conclude, as acknowledged in [11], that such spiral arm models are an overly simplistic representation of the real picture. The results suggest that the significant clustering that we measured between HMXBs and OBAs is due essentially to the physical locations of the individual objects in space, as opposed to being a shared attribute of the overall populations, e.g., that they appear to trace the spiral arms, or that they seem to follow a Gaussian distribution, etc. 

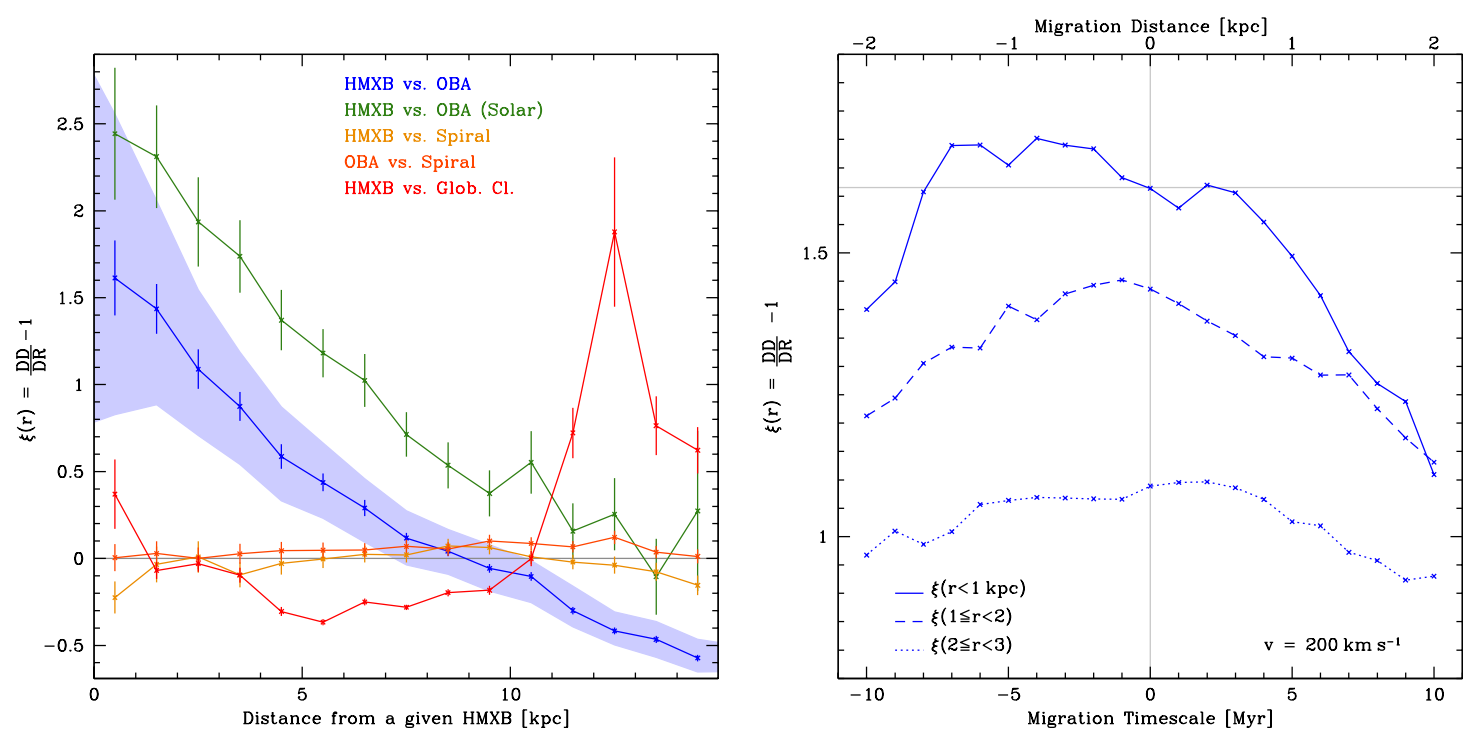

Figure 2: Left: The spatial cross-correlation function $\xi$ (blue curve) shows a significant $(\gtrsim 7 \sigma)$ deviation from 0 (Poisson statistics) at short distances from an HMXB, suggesting that its neighbors tend to be OBAs in their observed distribution rather than OBAs drawn from a catalog in which they are distributed randomly. The shaded area shows the $3 \sigma$ region from reshuffling the locations of HMXBs within their reported lineof-sight distance uncertainties. Extinction has a negligible effect as demonstrated by the persistence of the clustering signal when only objects in the Solar vicinity are considered (green curve). The HMXBs and OBAs are not clustered with the spiral arms (yellow and orange curves), nor are HMXBs clustered with an unlikely parent population such as globular clusters (green curve). Right: The values of $\xi$ resulting from shifting the positions of the HMXBs along their Galactic orbits. The shifts correspond to 1-10 Myr (in steps of $1 \mathrm{Myr}$ ) of circular motion at a velocity of $200 \mathrm{~km} \mathrm{~s}^{-1}$ in the forward (+) and reverse (-) directions of time. The continuous curve represents the values for $\xi(r<1 \mathrm{kpc})$, the dashed curve is $\xi(1 \leq r<2)$, and the dotted curve is $\xi(2 \leq r<3)$.

Clustering between HMXBs and OB associations is expected. How would $\xi$ react if HMXBs were compared to a population of sources for which no spatial correlation is expected? We tested the correlation functions of HMXBs against a set of 133 globular clusters from [13] that are located less than $20 \mathrm{kpc}$ from the GC. Globular clusters contain older populations such as cool KM dwarf stars and low-mass X-ray binaries (LMXBs). Unlike HMXBs, globular clusters (and LMXBs) are densely packed in the Galactic Bulge and their numbers drop exponentially with increasing radius from the GC. The random distribution of globular clusters was thus modeled as an exponential decay law to mimic the observed distribution. Figure 2 shows that $\xi$ between HMXBs and globular clusters is consistent with 0 for $r<1 \mathrm{kpc}$ from an HMXB. For any given HMXB, its immediate neighbors were just as likely to be drawn from the observed set as from the random set. In other words, $\xi$ reflects the expected lack of clustering between these unrelated stellar populations.

\section{Discussion}

There are several questions that we seek to address using $\xi$. What is the characteristic scale of the clustering between HMXBs and OBAs? Can this scale be used to constrain the amount of 
migration due to the runaway velocity imparted to the system during and after the formation of the compact object? Overall, what can this scale tell us about the evolutionary history of HMXBs?

\subsection{Migration due to Galactic rotation}

Outside of the inner Galaxy (i.e., galactocentric radius $R \gtrsim 2 \mathrm{kpc}$ ), objects orbit around the GC at a velocity of $\sim 200 \mathrm{~km} \mathrm{~s}^{-1}$. Thus, a star at the Solar galactocentric radius $(7.6 \mathrm{kpc})$ will have moved $6.3 \times 10^{15} \mathrm{~km}(200 \mathrm{pc})$ in $1 \mathrm{Myr}$ (defined as the migration timescale or $\tau$ ), assuming a circular orbit. In this manner, we rotated the HMXBs in space along their galactocentric orbits corresponding to $\tau=1-10 \mathrm{Myr}$ (in steps of $1 \mathrm{Myr}$ ) of circular motion in both the forward (+) and reverse $(-)$ directions of time (clockwise and counter-clockwise, respectively, in Fig. 1). Figure 1 presents the values of $\xi$ resulting from these shifts for small radii ( $r<3 \mathrm{kpc}$ from an HMXB).

If HMXB migration were related solely to Galactic rotation, then we would expect to see the amplitude of $\xi$ maximized for migration timescales around $-10 \mathrm{Myr}$. Instead, we find that the amplitude of $\xi$ is maximized for migration timescales between -2 and -7 Myr. So Galactic rotation can not fully explain the behavior of $\xi$ under different migration timescales.

\subsection{Migration due to the "slingshot" effect}

One mechanism suspected of giving an HMXB a significant velocity with respect to its parent OBA is the "slingshot" effect. This runaway velocity is the result of recoil (i.e., conservation of momentum) due to anisotropic mass loss from the newly-formed compact object to its companion star [14]. The magnitude and direction of this runaway velocity can be derived from the radial velocity or proper motion when the identity of its parent $\mathrm{OB}$ association and the line-of-sight distances are known. The HMXB can then be traced back to its birthplace leading to an estimation of its kinematical age.

We emphasize that there is no clearly-identified OB association for most HMXBs in our sample. Yet the vestiges of this dynamic history are imprinted on the overall HMXB distribution. Consider the distance separating each HMXB from its nearest OBA [16]. Excluding separations larger than $1 \mathrm{kpc}$, because they are likely too large to be due to runaway velocities, we obtain an average minimum distance of $r_{\min }=0.4 \pm 0.2 \mathrm{kpc}$ between an HMXB and an OBA. With this $r_{\min }$, and with $\xi$ maximized at $\tau \sim-4 \mathrm{Myr}$ (Fig. 2), we estimate an average runaway velocity of $100 \pm 50 \mathrm{~km} \mathrm{~s}^{-1}$ for the HMXBs in our sample. This is consistent with measurements of the runaway velocities in individual objects $[17,18]$.

Alternatively, with $r_{\min }=0.4 \pm 0.2 \mathrm{kpc}$ and assuming $v=100 \mathrm{~km} \mathrm{~s}^{-1}$, this translates to a migration timescale of $4 \pm 2 \mathrm{Myr}$ which is consistent with theoretical predictions for the average kinematical age of runaway massive binaries $[14,19]$. Notice that these kinematical age and distance scales are consistent with the distribution of $\xi$ whose value is maximized between -2 and $-7 \mathrm{Myr}$ (Fig. 2). The range of shifts that increase the correlation amplitude is wide reflecting the broad parameter space of velocities and kinematical ages represented within the HMXB class. Dynamical ejection from the cluster prior to the supernova phase can lead to runaway velocities of the order of 150-200 $\mathrm{km} \mathrm{s}^{-1}[20,21,22]$. This would make it difficult to retrace the trajectory of the HMXB back to its parent association, and the migration distance would be larger than expected from the runaway velocity alone. This is another factor contributing to the wide range in the shifts 
that increase the amplitude of $\xi$. Thus, the observed distribution of HMXBs in the Milky Way is consistent with the view that these systems have high velocities, on average.

Minimum separation distances can provide clues to the HMXB-OBA connection in specific cases. For example, there are $8 \mathrm{HMXBs}$ in our sample for which the uncertainty on the line-of-sight distance is smaller than the distance separating it from its nearest OBA. Those with separations smaller than the average minimum separation distance $(\sim 0.4 \mathrm{kpc})$ are prime examples of HMXBs whose migration distances are consistent with those expected from the slingshot effect. Small separation distances can result from runaway velocities that are lower than average, which can occur during the formation of a black hole since less material is expected to be expelled during the supernova (or gamma-ray burst) that created it [23].

A separation distance larger than $0.4 \mathrm{kpc}$ could imply that the binary inherited a higher runaway velocity than average, or that it experienced dynamical ejection prior to its supernova (placing the system further away from its OBA than would be expected from its velocity and kinematical age). Another possibility is that the OBA that produced the HMXB is no longer active enough to be catalogued: massive stars can photo-evaporate their molecular clouds on timescales of $30 \mathrm{Myr}$ [24].

On the other hand, if the HMXB distance is relatively accurate (i.e., within $\pm 0.5 \mathrm{kpc}$ ), and if the distance to the nearest OBA is less than this uncertainty, then this increases the likelihood that the objects were linked in the past. This is the case for $12 \mathrm{HMXBs}$ in our sample. This assumes (perhaps unjustifiably) that the nearest OBA is the most likely birthplace of the HMXB being considered.

\section{Conclusions}

We have demonstrated, for the first time, a significant clustering of HMXBs and OBAs in the Milky Way. Since the correlation function relates objects in Cartesian space (adding a third dimension is trivial), this is a more robust spatial relation than those derived in prior studies which focused on the distribution of a single dimension such as longitudes or galactocentric radii.

Not only does the correlation function confirm the expected view that HMXBs and OBAs are clustered together, it shows that the populations are not perfectly aligned. There exists a slight offset between the two populations whose characteristic scale contains the vestiges of stellar and Galactic evolution. Thanks to the correlation function, the average runaway velocity gained by an HMXB after the supernova can be constrained to $100 \pm 50 \mathrm{~km} \mathrm{~s}^{-1}$. This translates to kinematical ages (time spanning the supernova and HMXB phases) of $4 \pm 2 \mathrm{Myr}$, which is consistent with theoretical expectations. We point out that these results are based on the distributions of the HMXB and OBA populations which compose the "grand design" of the Milky Way. Yet even with this global view, the correlation function allows us to deduce the effects of local perturbations such as runaway velocities.

The correlation function opens novel areas of research both inside the Galaxy (e.g., more accurate spiral arm models are possible with this analysis technique) and beyond (e.g., comparing the populations of HMXBs and OBAs in other nearby galaxies). An increase in the discovery space of HMXB populations, thanks to ongoing surveys by INTEGRAL, should allow us to probe deeper into the evolutionary history of massive stars and compact objects. This will permit a better 
understanding of the stellar content and its distribution in the Galaxy. These results were published as Bodaghee et al. (2012), ApJ, 744, 108 [25].

\section{References}

[1] Schaller, G., Schaerer, D., Meynet, G., \& Maeder, A. 1992, A\&AS, 96, 269

[2] Bronfman, L., Casassus, S., May, J., \& Nyman, L. 2000, A\&A, 358, 521

[3] Mirabel, I. F., Rodrigues, I., \& Liu, Q. Z. 2004, A\&A, 422, L29

[4] Grimm, H., Gilfanov, M., \& Sunyaev, R. 2002, A\&A, 391, 923

[5] Lutovinov, A., Revnivtsev, M., Gilfanov, M., Shtykovskiy, P., Molkov, S., \& Sunyaev, R. 2005, A\&A, 444, 821

[6] Dean, A.J., Bazzano, A., Hill, A.B., Stephen, J.B., Bassani, L., Barlow, E., Bird, A.J., Lebrun, F., Sguera, V., Shaw, S.E., Ubertini, P., Walter, R., \& Willis, D.R. 2005, A\&A, 443, 485

[7] Bodaghee, A., Courvoisier, T.J.-L., Rodriguez, J., Beckmann, V., Produit, N., Hannikainen, D., Kuulkers, E., Willis, D.R., \& Wendt, G.E., 2007, A\&A, 467, 585

[8] Lutovinov, A., Revnivtsev, M., Gilfanov, M., \& Sunyaev, R. 2008, [astro-ph / 0801 . 3589]

[9] Peebles, P. J. E. 1980, The large-scale structure of the universe, Princeton University Press, 435

[10] Landy, S. D., \& Szalay, A. S. 1993, ApJ, 412, 64

[11] Russeil, D. 2003, A\&A, 397, 133

[12] Vallée, J.P. 2008, ApJ, 681, 303

[13] Bica, E., Bonatto, C., Barbuy, B., \& Ortolani, S. 2006, A\&A, 450, 105

[14] Blaauw, A. 1961, Bull. Ast. Inst. Netherlands, 15, 265

[15] Brandt, N., \& Podsiadlowski, P. 1995, MNRAS, 274, 461

[16] van den Heuvel, E. P. J., Portegies Zwart, S. F., Bhattacharya, D., \& Kaper, L. 2000, A\&A, 364, 563

[17] Gunn, J. E., \& Ostriker, J. P. 1970, ApJ, 160, 979

[18] Stone, R. C. 1979, ApJ, 232, 520

[19] van Oijen, J. G. J. 1989, A\&A, 217, 115

[20] Poveda, A., Ruiz, J., \& Allen, C. 1967, Boletin de los Observatorios Tonantzintla y Tacubaya, 4, 86

[21] Gies, D. R., \& Bolton, C. T. 1986, ApJS, 61, 419

[22] Pflamm-Altenburg, J., \& Kroupa, P. 2010, MNRAS, 404, 1564

[23] Mirabel, I. F., \& Rodrigues, I. 2003, Science, 300, 1119

[24] Williams, J. P., \& McKee, C. F. 1997, ApJ, 476, 166

[25] Bodaghee, A., Tomsick, J.A., Rodriguez, J., \& James, J.B. 2012, ApJ, 744, 108 